\title{
Target Deconvolution of Fenofibrate in Nonalcoholic Fatty Liver Disease Using Bioinformatics Analysis
}

\author{
Ali Mahmoudi, ${ }^{1}$ Alexandra E. Butler, ${ }^{2}$ Tannaz Jamialahmadi, ${ }^{3}$ \\ and Amirhossein Sahebkar $\mathbb{D I}^{4,5,6}$ \\ ${ }^{1}$ Department of Medical Biotechnology and Nanotechnology, Faculty of Medicine, Mashhad University of Medical Sciences, Iran \\ ${ }^{2}$ Royal College of Surgeons in Ireland Bahrain, Adliya, Bahrain \\ ${ }^{3}$ Department of Nutrition, Faculty of Medicine, Mashhad University of Medical Sciences, Mashhad, Iran \\ ${ }^{4}$ Biotechnology Research Center, Pharmaceutical Technology Institute, Mashhad University of Medical Sciences, Mashhad, Iran \\ ${ }^{5}$ Applied Biomedical Research Center, Mashhad University of Medical Sciences, Mashhad, Iran \\ ${ }^{6}$ School of Pharmacy, Mashhad University of Medical Sciences, Mashhad, Iran
}

Correspondence should be addressed to Amirhossein Sahebkar; amir_saheb2000@yahoo.com

Received 22 October 2021; Revised 12 November 2021; Accepted 14 December 2021; Published 26 December 2021

Academic Editor: Bing Niu

Copyright ( $\odot 2021$ Ali Mahmoudi et al. This is an open access article distributed under the Creative Commons Attribution License, which permits unrestricted use, distribution, and reproduction in any medium, provided the original work is properly cited.

Background. Nonalcoholic fatty liver disease (NAFLD) is a prevalent form of liver damage, affecting 25\% of the global population. NAFLD comprises a spectrum of liver pathologies, from hepatic steatosis to nonalcoholic steatohepatitis (NASH), and may progress to liver fibrosis and cirrhosis. The presence of NAFLD correlates with metabolic disorders such as hyperlipidemia, obesity, blood hypertension, cardiovascular, and insulin resistance. Fenofibrate is an agonist drug for peroxisome proliferator-activated receptor alpha $(\operatorname{PPAR} \alpha)$, used principally for treatment of hyperlipidemia. However, fenofibrate has recently been investigated in clinical trials for treatment of other metabolic disorders such as diabetes, cardiovascular disease, and NAFLD. The evidence to date indicates that fenofibrate could improve NAFLD. While PPAR $\alpha$ is considered to be the main target of fenofibrate, fenofibrate may exert its effect through impact on other genes and pathways thereby alleviating, and possibly reversing, NAFLD. In this study, using bioinformatics tools and gene-drug, gene-diseases databases, we sought to explore possible targets, interactions, and pathways involved in fenofibrate and NAFLD. Methods. We first determined significant protein interactions with fenofibrate in the STITCH database with high confidence (0.7). Next, we investigated the identified proteins on curated targets in two databases, including the DisGeNET and DISEASES databases, to determine their association with NAFLD. We finally constructed a Venn diagram for these two collections (curated genesNAFLD and fenofibrate-STITCH) to uncover possible primary targets of fenofibrate. Then, Gene Ontology (GO) and KEGG were analyzed to detect the significantly involved targets in molecular function, biological process, cellular component, and biological pathways. A $P$ value $<0.01$ was considered the cut-off criterion. We also estimated the specificity of targets with NAFLD by investigating them in disease-gene associations (STRING) and EnrichR (DisGeNET). Finally, we verified our findings in the scientific literature. Results. We constructed two collections, one with 80 protein-drug interactions and the other with 95 genes associated with NAFLD. Using the Venn diagram, we identified 11 significant targets including LEP, SIRT1, ADIPOQ, PPARA, SREBF1, LDLR, GSTP1, VLDLR, SCARB1, MMP1, and APOC3 and then evaluated their biological pathways. Based on Gene Ontology, most of the targets are involved in lipid metabolism, and KEGG enrichment pathways showed the PPAR signaling pathway, AMPK signaling pathway, and NAFLD as the most significant pathways. The interrogation of those targets on authentic disease databases showed they were more specific to both steatosis and steatohepatitis liver injury than to any other diseases in these databases. Finally, we identified three significant genes, APOC3, PPARA, and SREBF1, that showed robust drug interaction with fenofibrate. Conclusion. Fenofibrate may exert its effect directly or indirectly, via modulation of several key targets and pathways, in the treatment of NAFLD. 


\section{Introduction}

In recent decades, nonalcoholic fatty liver (NAFLD) has received greater attention from both healthcare professionals and the general public due to its increasing prevalence. NAFLD comprises a spectrum of liver disorders, from hepatic steatosis to nonalcoholic steatohepatitis (NASH) and, if unchecked, may progress to fibrosis and cirrhosis [1]. The hallmark of NAFLD is accumulation of fat deposits in hepatocytes, the presence of which correlates with metabolic disorders such as hyperlipidemia, obesity, hypertension, cardiovascular disease, and insulin resistance $[2,3]$. The global prevalence of NAFLD is approximately $\sim 25 \%$, with the highest prevalence being found in the Middle East [4].

Despite the health burden it imposes, no definitive treatment for NAFLD has yet been determined, though various therapeutic approaches have been proposed. Lifestyle intervention and pharmacological interventions are the mainstays of treatment for patients with NAFLD.

As disruption of essential genes and proteins may lead to fatty liver diseases, identification of these targets enables drug discovery for treatment of NAFLD [5, 6]. Drug treatments include targeting caloric intake and disposal, inflammation, lipotoxicity, and cirrhosis [7-10]. One of the key drug targeting strategies is the modulation of hepatic fat accumulation, including targeting peroxisome proliferatoractivator receptors and de novo lipogenesis [11]. Fenofibrate is an agonist drug for $\operatorname{PPAR} \alpha$ and is principally used for treatment of hyperlipidemia in spite of the presence of statins and several newer lipid-lowering agents [12-15]. $\operatorname{PPAR} \alpha$ is abundantly expressed in the liver and modulates various genes implicated in the catabolism of fatty acids [16]. However, it has recently been investigated in clinical trials for treatment of other metabolic disorders such as diabetes, cardiovascular disease, and NAFLD [17-19]. Additionally, reports suggest that fenofibrate could play a role in antioxidation, tumour apoptosis, anti-inflammation, and antifibrosis plus several other pliotropic effects [20-28]. Evidence from several clinical studies has shown that fenofibrate may provide benefit to patients with NASH/NAFLD $[18,29,30]$.

While $\operatorname{PPAR} \alpha$ is recognized to be the main target of fenofibrate, this drug may exert its effect via other genes and pathways that have not been well characterized to improve and possibly reverse NAFLD/NASH. Clinical investigation is already underway using fenofibrate for the treatment of NAFLD. Using bioinformatics tools and genedrug, gene-diseases databases, we sought to explore other targets, interactions, and pathways involving fenofibrate and NAFLD. In Figure 1, we illustrate the overall strategy employed in this study.

\section{Methods}

2.1. Fenofibrate and Target Search. We first searched interactions of fenofibrate in the STITCH database (http://stitch .embl.de/) to explore essential protein targets. STITCH is a platform for diagnosis interaction between chemicals and proteins. Here, we considered the high confidence cut-off (0.700) and limited species only to Homo sapiens.

\subsection{Exploring Important NAFLD Genes in DISEASES and} DisGeNET Databases. Next, we investigated the protein targets identified in STITCH on curated targets in two databases, the DisGeNET database (https://www.disgenet.org/ (and the DISEASES database (http://diseases.jensenlab.org/), to find their association with NAFLD. DisGeNET is a database that contains a collection of genes associated with specific diseases. That data is integrated from a variety of sources such as expert-curated repositories, the scientific literature and GWAS catalogs. DisGeNET currently covers more than 1,700 genes and 24,000 diseases and traits [31]. For association genes with NAFLD, 1,058 genes were registered in this database. Curated data contain seven primary resources: UNIPROT, ORPHANET, CTD, GENOMICS ENGLAND, CLINGEN, PSYGENET, and CGI. To achieve a curated dataset from DisGeNET, we used a plugin in the cystoscope to construct curated sources targets for NAFLD. DISEASES database is a weekly updated database that comprises diseases and gene relations from different resources, including manually curated literature, text mining, cancer mutation data, and genome-wide association research [32]. We extracted the targets from the available resources, including experiments and manually curated literature associated with NAFLD.

2.3. Venn Diagram to Obtain Important Fenofibrate Interaction Protein Targets in NAFLD. We finally created a Venn diagram (http://bioinformatics.psb.ugent.be/webtools/ Venn/) for these two collections (curated genes-NAFLD and fenofibrate-STITCH) to find important targets of fenofibrate beyond the conventionally recognized targets.

2.4. Gene Ontology Pathway Enrichment Analyses for Target Proteins of Fenofibrate. Gene ontology (GO) enrichment is a popular procedure used to interpret genes and stratify them in three major categories, those that contribute to molecular function (MF), biological process, (BP) or cellular component (CC). GO was analyzed for important targets obtained from the Venn diagram using the Gene Ontology resource with the web address: http://geneontology.org. Additionally, KEGG was analyzed using the Enrichr database with the web address: https://maayanlab.cloud/Enrichr/. The KEGG pathway is a comprehensive database that maps pathways according to their metabolic interrelationships. In GO and KEGG analyses, the $P$ value $<0.01$ was considered the cutoff criterion. We also analyzed the enrichment pathways using the wikipathways plugin in Cytoscape version 3.8.2. A $P$ value $<0.01$ was considered the cut-off criterion. In addition, we estimated the specificity of obtained targets with NAFLD and investigated them in disease-gene associations (STRING) and EnrichR (DisGeNET).

\section{Results}

3.1. Protein Target Interaction with Fenofibrate in the STITCH Database. Screening fenofibrate in the STITCH database with high confidence (0.7) identified 80 protein 


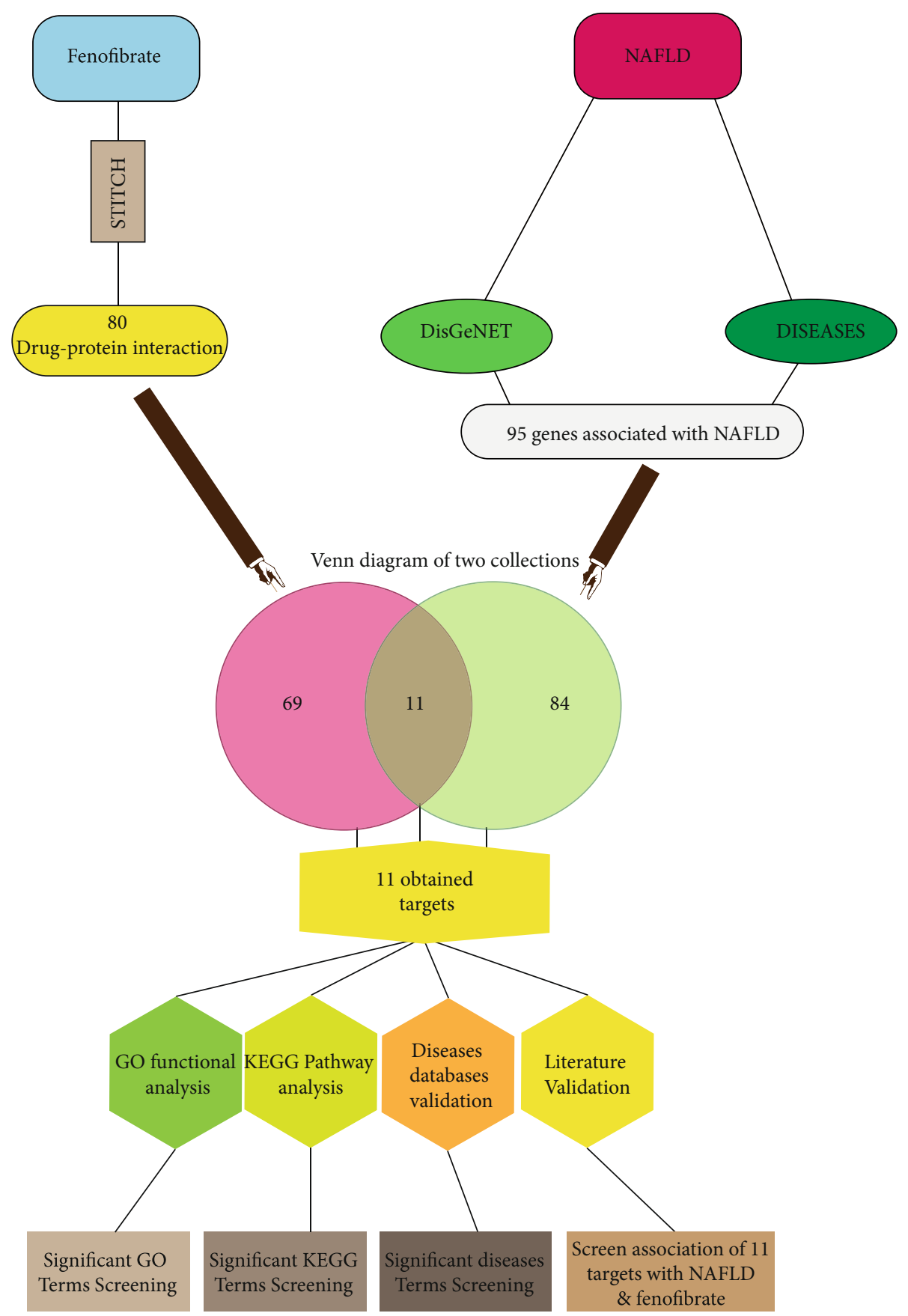

FIGURE 1: A comprehensive diagram illustrating the investigative strategy undertaken in the present study.

targets. The drug-protein interaction was visualized on Cytoscape (Figure 2(a)).

3.2. Discovering Curated NAFLD Genes. The curated data DisGeNet plugin on Cytoscape and DISEASES database identified 95 genes associated with NAFLD. All the data are visualized with Cytoscape software (Figure 2(a)).

3.3. Overlap of Fenofibrate Targets on the STITCH and Curated NAFLD Genes Visualized Using a Venn Diagram. A Venn diagram of the two created datasets revealed eleven candidates, including SREBF1, SCARB1, LDLR, PPARA,
VLDLR, LEP, MMP1, GSTP1, SIRT1, APOC3, and ADIPOQ (Figure 2(b)) that may be directly or indirectly affected by fenofibrate. The scoring based on DisGeNET is shown in Table 1. Based on the database algorithm (genes-disease associate score), five targets (LEP, SIRT1, ADIPOQ, PPARA, and SREBF1) are the most important in NAFLD.

3.4. GO and KEGG Enrichment Analyses of Protein Targets of Fenofibrate. GO analysis of the 11 identified protein targets demonstrated major involvement in the regulation of the lipid biosynthetic process, the lipid metabolic process, and the lipid metabolic process under biological process 


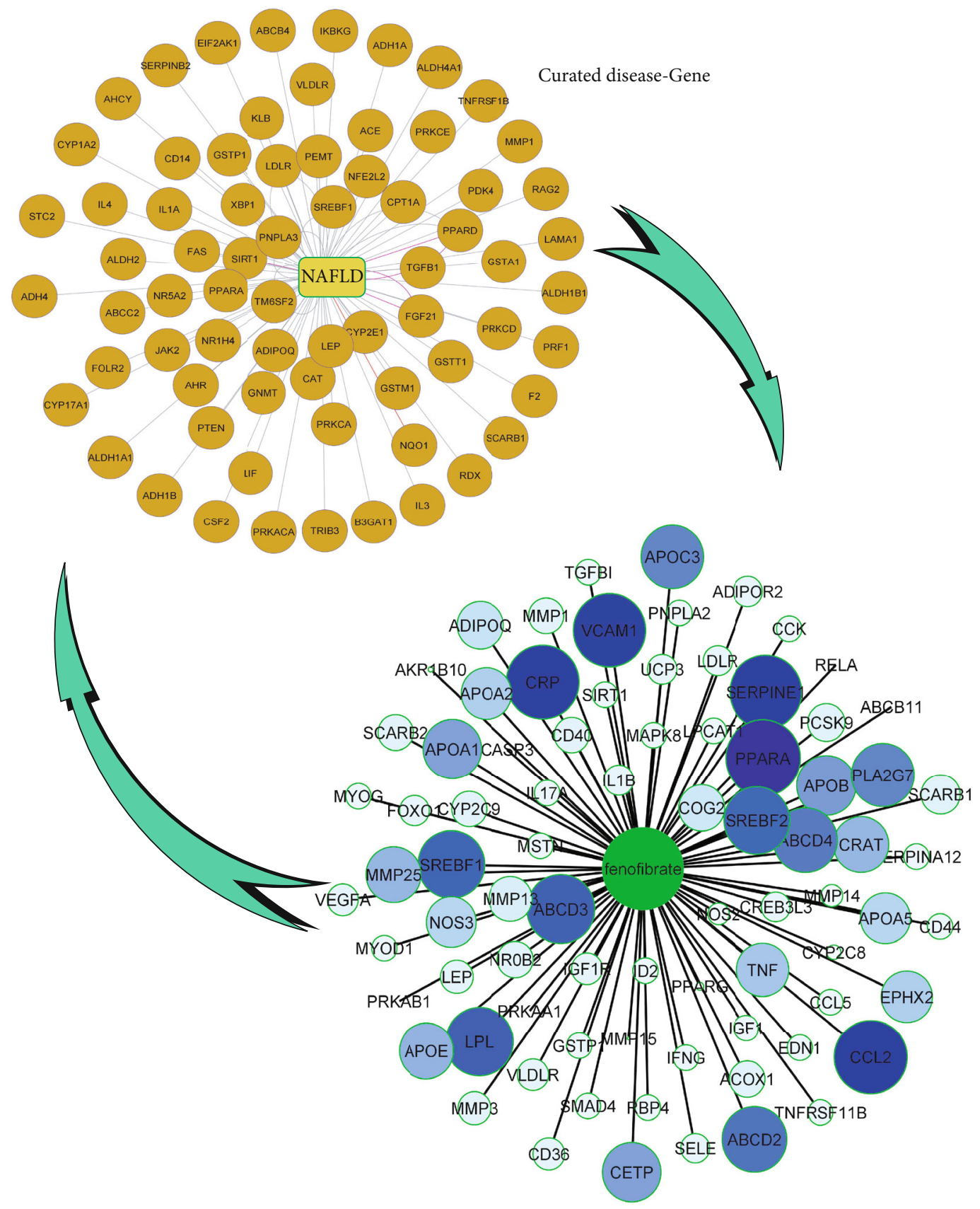

Fenofibrate-STITCH Network

(a)

Figure 2: Continued. 


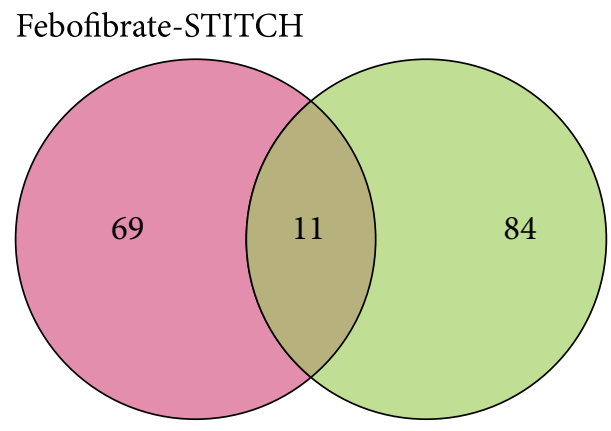

Curated gene-disease

(b)

FIGURE 2: (a) Curated disease-gene database and fenofibrate-protein interaction visualized with Cytoscape software. (b) Venn diagram of the two datasets comprising curated disease-gene database and fenofibrate-protein interaction.

TABLE 1: The relationship of genes associated with NAFLD that are targets of fenofibrate (http://www.disgenet.org/).

\begin{tabular}{|c|c|c|c|c|c|c|}
\hline Gene & UniProt & Gene full name & Protein class & $\begin{array}{c}\text { DSI } \\
\mathrm{g}\end{array}$ & $\begin{array}{c}\text { Score } \\
\text { gda }\end{array}$ & $\begin{array}{c}\text { STITCH } \\
\text { score }\end{array}$ \\
\hline LEP & P41159 & Leptin & Plasma protein & 0.349 & 0.4 & 0.829 \\
\hline SIRT1 & Q96EB6 & Sirtuin 1 & Epigenetic regulator & 0.378 & 0.4 & 0.8 \\
\hline ADIPOQ & Q15848 & $\begin{array}{c}\text { Adiponectin, } \mathrm{C} 1 \mathrm{Q} \text {, and collagen domain } \\
\text { containing }\end{array}$ & Transporter & 0.376 & 0.4 & 0.884 \\
\hline PPARA & Q07869 & Peroxisome proliferator-activated receptor alpha & Nuclear receptor & 0.432 & 0.4 & 0.995 \\
\hline SREBF1 & P36956 & $\begin{array}{l}\text { Sterol regulatory element binding transcription } \\
\text { factor } 1\end{array}$ & $\begin{array}{l}\text { Plasma protein, transcription } \\
\text { factor }\end{array}$ & 0.518 & 0.38 & 0.962 \\
\hline$L D L R$ & $\mathrm{P} 01130$ & Low-density lipoprotein receptor & Plasma protein & 0.449 & 0.37 & 0.816 \\
\hline GSTP1 & P09211 & Glutathione S-transferase pi 1 & Enzyme & 0.383 & 0.33 & 0.8 \\
\hline$V L D L R$ & P98155 & Very-low-density lipoprotein receptor & Plasma protein & 0.558 & 0.31 & 0.823 \\
\hline$S C A R B 1$ & Q8WTV0 & Scavenger receptor class B member 1 & Receptor & 0.559 & 0.3 & 0.853 \\
\hline$M M P 1$ & P03956 & Matrix metallopeptidase 1 & Enzyme & 0.385 & 0.3 & 0.872 \\
\hline APOC3 & P02656 & Apolipoprotein C3 & Plasma protein & 0.531 & 0.1 & 0.944 \\
\hline
\end{tabular}

Score gda (genes-disease associate score): the gda score is based on supporting evidence that has been collated from different sources as regards the association of genes and diseases. The gda score ranges from 0 to 1 , so the closer this range is to 1 indicates a stronger the association between gene and disease. DSI (disease specificity index): a gene may be associated with numerous diseases. This index indicates the specificity of diseases to a particular gene. The index ranges from 0 to 1 . A gene with many disease associations has a zero DSI index and, by contrast, a gene associated with just one disease has a DSI of 1 . STITCH score: the STITCH score is a confidence indicator of how likely it is that STITCH will correctly evaluate an interaction based on evidence from preceding studies and predictions. Scores range from 0 to 1 , with one being the highest confidence level for interaction and 0 being the highest level of uncertainty. A score of 0.5 , for example, indicates that the interaction could be $50 \%$ false (i.e., a false positive).

(Table 2). This analysis additionally showed that these protein targets were chiefly involved in lipoprotein particle receptor activity, protein-lipid complex binding, and lipoprotein particle binding under the molecular function category. Furthermore, cellular components included the lipoprotein particle, plasma lipoprotein particle, and protein-lipid complex (Table 2).

In KEGG enrichment, we observed several biological pathways. The highest $P$ value pathways included the PPAR signaling pathway and the AMPK signaling pathway (Table 3).

The DISEASES database, based on the STRING algorithm, and the DisGeNETdatabase, based on the EnrichR algorithm, revealed the association of 11 of the identified proteins with NAFLD and its advanced form NASH, these diseases ranking in the top three in both databases
(Table 4). Therefore, their close relationship with fatty liver disease was confirmed.

In contrast, by setting the cut-off for the STITCH database to 0.9 , we identified 22 protein interactions with fenofibrate. PPARA, with a score of 0.995 , was the highest ranked protein target in this PPI network. SERPINE1, CCL2, CRP, and VCAM1, with a score of 0.984, were the next most significant protein targets after PPARA in the PPI network. Moreover, protein targets were restricted to three significant genes in the Venn diagram with a preprepared curated disease database. Those three protein targets were SREBF1, APOC3, and PPARA.

We also visualized the degree of connection with high confidence (0.7) of the protein targets to fenofibrate in the NAFLD pathway. The more intense color indicates greater interaction (based on the STITCH score) (Figure 3). The 
TABLe 2: Gene ontology enrichment analysis via Enrichr for the 11 identified genes with the best score interaction with fenofibrate.

(a) Biological process (GO)

\begin{tabular}{|c|c|c|c|c|}
\hline Accession & Pathway description & Gene count & $P$ value & FDR \\
\hline GO:0046890 & Regulation of lipid biosynthetic process & 7 & $7.07 E^{-13}$ & $1.11 E^{-08}$ \\
\hline GO:0019216 & Regulation of lipid metabolic process & 8 & $7.10 E^{-13}$ & $5.59 E^{-09}$ \\
\hline GO:0034381 & Llasma lipoprotein particle clearance & 5 & $1.76 E^{-12}$ & $9.25 E^{-09}$ \\
\hline GO:0006629 & Lipid metabolic process & 10 & $3.39 E^{-12}$ & $1.33 E^{-08}$ \\
\hline GO:1905952 & Regulation of lipid storage & 5 & $6.75 E^{-11}$ & $2.13 E^{-07}$ \\
\hline
\end{tabular}

(b) Molecular function (GO)

\begin{tabular}{|c|c|c|c|c|}
\hline & Pathway description & Gene count & $P$ value & FDR \\
\hline GO:0030228 & Lipoprotein particle receptor activity & 3 & $1.50 E^{-07}$ & $7.32 E^{-04}$ \\
\hline GO:0044877 & Protein-lipid complex binding & 3 & $5.57 E^{-07}$ & $1.36 E^{-03}$ \\
\hline GO:0071813 & Lipoprotein particle binding & 3 & $5.57 E^{-07}$ & $9.07 E^{-04}$ \\
\hline GO:0030229 & Very-low-density lipoprotein particle receptor activity & 2 & $3.88 E^{-06}$ & $4.75 E^{-03}$ \\
\hline GO:0005041 & Cargo receptor activity & 3 & $1.50 E^{-07}$ & $7.32 E^{-04}$ \\
\hline
\end{tabular}

(c) Cellular component (GO)

\begin{tabular}{|c|c|c|c|c|}
\hline & Pathway description & Gene count & $P$ value & FDR \\
\hline GO:1990777 & Lipoprotein particle & 3 & $1.11 E^{-06}$ & $2.20 E^{-03}$ \\
\hline GO:0034358 & Plasma lipoprotein particle & 3 & $1.11 E^{-06}$ & $1.10 E^{-03}$ \\
\hline GO:0032994 & Protein-lipid complex & 3 & $1.38 E^{-06}$ & $9.16 E^{-04}$ \\
\hline GO:0034361 & Very-low-density lipoprotein particle & 2 & $5.95 E^{-05}$ & $2.96 E^{-02}$ \\
\hline GO:0034385 & Triglyceride-rich plasma lipoprotein particle & 2 & $6.51 E^{-05}$ & $2.59 E^{-02}$ \\
\hline
\end{tabular}

FDR (false discovery rate): FDR is a stringent statistical method allowing multiple comparisons while preserving a low false-positivity rate.

TABLE 3: KEGG pathways for 11 critical genes interact with fenofibrate.

\begin{tabular}{|c|c|c|c|c|}
\hline Num. & Pathway name & $\begin{array}{l}\text { KEGG } \\
\text { Genes }\end{array}$ & Gene count & $P$ value \\
\hline 1 & PPAR signaling pathway & MMP1, ADIPOQ, APOC3, PPARA & 4 & $5.586 e-8$ \\
\hline 2 & AMPK signaling pathway & SREBF1, LEP, ADIPOQ, SIRT1 & 4 & $3.937 e-7$ \\
\hline 3 & Nonalcoholic fatty liver disease & SREBF1, LEP, ADIPOQ, PPARA & 4 & 0.000001098 \\
\hline 4 & Cholesterol metabolism & SCARB1, APOC3, LDLR & 3 & 0.000002392 \\
\hline 5 & Adipocytokine signaling pathway & LEP, ADIPOQ, PPARA & 3 & 0.000006357 \\
\hline
\end{tabular}

pathways are constructed based upon the wikipathway dataset with access number WP4396 using the Cytoscape plugin.

\section{Discussion}

NAFLD is a highly prevalent chronic liver disease, comprising a spectrum of liver pathologies, from hepatic steatosis to nonalcoholic steatohepatitis (NASH), and may progress to liver fibrosis and cirrhosis. [33]. NAFLD is commonly identified as a multifactorial disease with interaction amongst risk factors and susceptibility genes that play a central role in the development and phenotype of NAFLD [34]. Conse- quently, identifying those targets and employing suitable therapeutic agents are important steps in improving treatment modalities. Fenofibrate is a drug that is proven for treatment of hyperlipidemia. Additionally, some studies have shown positive results with fenofibrate for the treatment of NAFLD. Fenofibrate has been shown to improve NAFLD in various research studies using cell lines and animal models, as well as in clinical studies in humans. For example, it has been reported that fenofibrate reduces fat content in the liver, reverses hepatic steatosis and fibrosis, and alleviates pathological liver changes in animals with NAFLD [35, 36]. A clinical study revealed that liver 
TABLE 4: Association of protein targets obtained in the interaction of fenofibrate with fatty liver disease in disease databases.

(a) Disease-gene associations (STRING)

\begin{tabular}{lcc}
\hline Identifier & Primary name & FDR \\
\hline DOID:0080208 & Nonalcoholic fatty liver disease & $4.23 e-07$ \\
DOID:11716 & Prediabetes syndrome & 0.0037 \\
DOID:0080547 & Nonalcoholic steatohepatitis & 0.0058 \\
\hline
\end{tabular}

(b) DisGeNET (EnrichR)

\begin{tabular}{lcc}
\hline & Primary name & $P$ value \\
\hline 1 & Nonalcoholic fatty liver disease & $1.598 e-17$ \\
2 & Nonalcoholic steatohepatitis & $6.748 e-17$ \\
3 & Acute coronary syndrome & $4.157 e-16$ \\
\hline
\end{tabular}

enzymes, blood pressure, and body mass index considerably improved, and that treatment with fenofibrate was advantageous due to its beneficial effects in patients with NAFLD [37]. Moreover, an in vitro study on steatotic HepaRG cell lines demonstrated the effect of fenofibrate on ameliorating hepatic steatosis [38]. The mechanism that has been widely purported is through fenofibrate's antagonistic effect on PPAR $\alpha$. However, in our study, according to the results of prediction and its interactions directly and indirectly with other targets using bioinformatics software, the mechanism of fenofibrate's mode of action may be broader than an exclusive role of PPAR $\alpha$ antagonism. Here, we investigated and analyzed those predictions in other disease-genes, drug-protein databases and biological pathways to understand in greater depth the possible effects of fenofibrate in NAFLD.

In the work presented here, we first searched significant prediction protein interaction with high confidence for fenofibrate. Then, we probed protein interaction in association with fatty liver disease and selected the most relevant targets. In so doing, we identified 11 significant targets, including LEP, SIRT1, ADIPOQ, PPARA, SREBF1, LDLR, GSTP1, VLDLR, SCARB1, MMP1, and APOC3, and we then evaluated their biological pathways. Based on gene ontology, most of the targets were involved in lipid metabolism, and KEGG enrichment pathways showed PPAR signaling pathway, AMPK signaling pathway, and NAFLD as the most significant pathways. The targets we found among the authentic disease databases were more specific to fatty liver disease (steatosis and steatohepatitis) than other diseases in these databases. We finally identified three important significant genes, APOC3, PPARA, and SREBF1, showing robust drug interaction with fenofibrate.

The PPAR signaling pathway is comprised of three receptor subtypes, alpha, gamma, and beta/delta, which are activated by fatty acids and their derivatives. Each subtype is encoded by a separate gene. PPAR-alpha is important for lipid metabolism in the liver and functions in the clearance of circulating and cellular lipids. The function of PPAR-gamma is in the induction of adipocyte differentia- tion which causes increased uptake of blood glucose. PPAR-beta/delta also contributes to lipid oxidation and cell proliferation $[39,40]$. By applying different agonists to this pathway, research has shown a decrease in triglycerides, modulation of circulating glucose, and an elevation in HDL [41-43]. In this way, these agonists could ameliorate NAFLD. The PPAR signaling pathway contains various genes that show enrichment in our study: based on KEGG analysis, enrichment of MMP1, ADIPOQ, APOC3, and PPARA revealed that this pathway shows a robust interaction with fenofibrate ( $P$ value: $5.586 e-8)$. PPAR-alpha is principally present in the liver, while PPAR-gamma is mainly expressed in adipose tissue [44]. Expression of PPAR-gamma has been reported to be significantly increased in the liver of patients and animal models with NAFLD [45-47]. This elevation might be a consequence of the expression of the adipogenic genes that induce lipid accumulation in the liver of these patients and animals with NAFLD [48].

AMPK (adenosine monophosphate-activated protein kinase) has serine/threonine kinase as its catalytic alpha subunit and beta/gamma as its regulatory subunit [49]. This pathway is involved in lipid metabolism and energy sensing, regulating glucose in numerous tissue such as the liver [50]. In our study, enrichment of SREBF1, LEP, ADIPOQ, and SIRT1 genes was found, and the AMPK pathway was significantly ( $P$ value: $3.937 e-7)$ shown to be influenced by fenofibrate. AMPK is regulated by phosphorylation and dephosphorylation via kinases. Activation of AMPK results in an orderly adjustment of energy balance in metabolic processes. It increases fatty acid oxidation and reduces triglyceride and cholesterol production, consequently decreasing fat accumulation [51]. Due to these actions, this pathway is considered to be a therapeutic target for a metabolic disorder such as NAFLD $[52,53]$. Activation of AMPK is connected to improvement in liver inflammation and metabolism in NAFLD [54, 55]. Additionally, several studies have demonstrated that AMPK signaling pathways are involved in liver steatosis and steatohepatitis [56]. Research in 2014 indicated that fenofibrate could modulate AMPK signaling and thereby exert its therapeutic effect [57].

Research has demonstrated that lipid accumulation in the liver involves a reduction in fatty acid oxidation and VLDL secretion and upregulation of adipogenic and lipogenic pathways, through which lipoprotein particles deliver fatty acids to the liver [58]. Based on the gene ontology analysis (Table 2), important genes in these pathways operate at the three stages of biological, molecular, and cellular components and could be influenced,with high confidence, by fenofibrate.

PPARA is one of the most important genes in NAFLD and that it interacts with fenofibrate is well established. As shown in our analysis, PPARA had the highest score for interaction with fenofibrate. Moreover, its role in NAFLD was also investigated, again showing a high score. However, our aim was to investigate targets other than PPARA and, hence, here, we discuss the possible influence of other targets with fenofibrate and their role in the pathogenesis of NAFLD. 


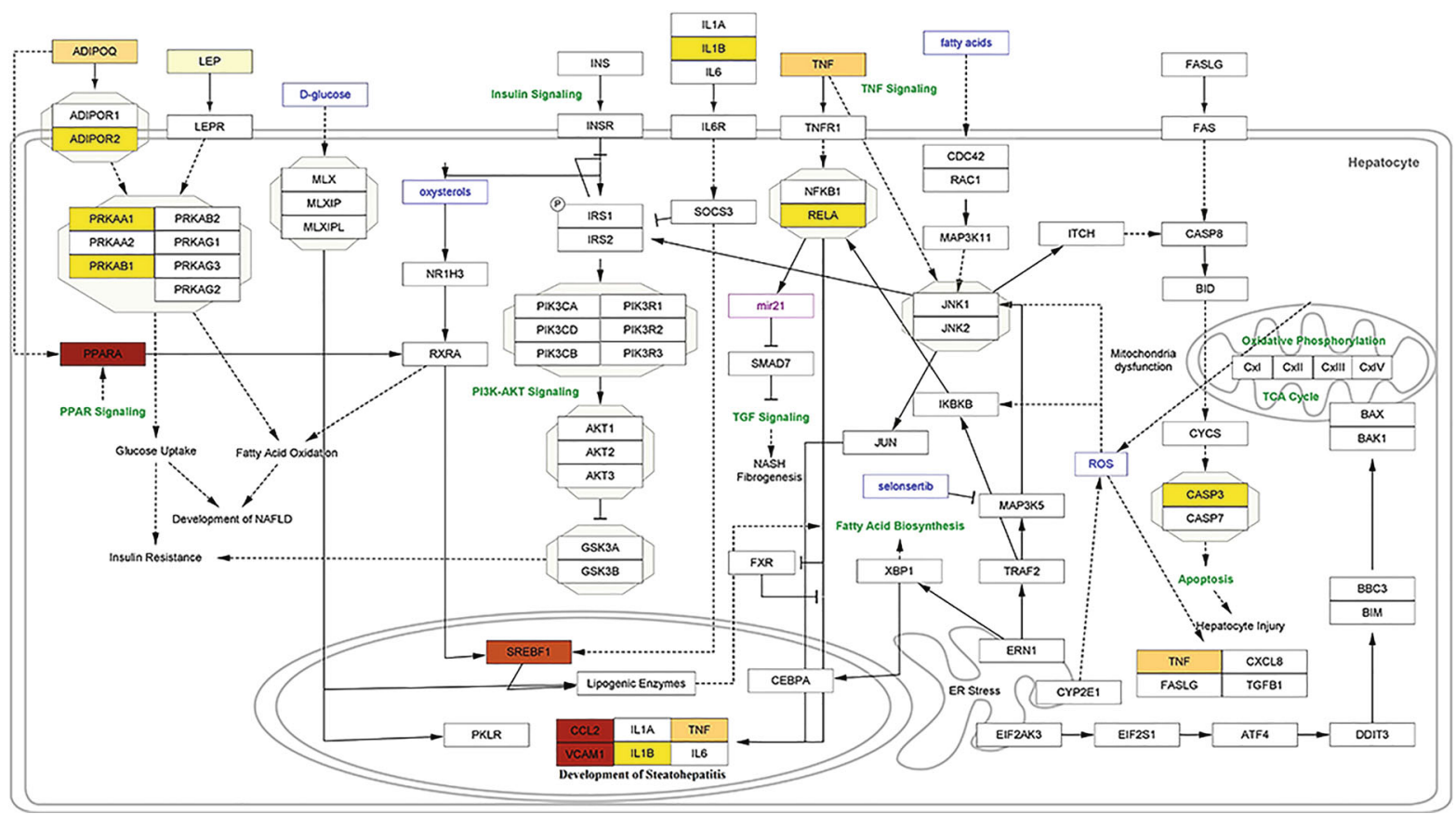

FIGURE 3: Visualizing protein interactions with fenofibrate in the NAFLD pathway with high confidence (0.7) based on the STITCH score. The intensity of color illustrates the degree of interaction of fenofibrate with the targets.

SREBF1 is an essential factor in modulation of lipogenesis [59]. In models of NAFLD, SREBF1 was downregulated following induction of steatosis [60]. However, in patients with NAFLD, SREBF1 was reported to be significantly higher than the control group [61]. Moreover, enhancing cleavage of SREBF2 was shown to boost lipogenesis [62]. A recent study revealed that SREBF1 is activated through zinc finger and BTB domain-containing 7A (ZBTB7A), which causes lipid accumulation and progression of NAFLD [63]. Numerous studies have reported that SREBF1 is a potential target influencing NAFLD, as evidenced by administration of various interval treatments [64-66]. A recent study in 2021 by Elsayed et al. indicated that SREBF1 elevation is a risk factor in the progression of NAFLD and that, following treatment of NAFLD, this gene was downregulated. Fenofibrate, by direct binding of PPARA to the DR1 motif of SREBF1, may induce SREBF1 expression [67]. A TRANSFAC analysis revealed that after treatment with fenofibrate, MuRF1 -/- genes commonly had a SREBF1 promoter region [68]. Other researchers showed that fenofibrate could promote CREBH products and reduce SREBF1 levels [69]. Of note, in our study (Table 1), SREBF1 had the second highest score for interaction with fenofibrate after PPARA and showed a strong relationship to NAFLD.

ADIPOQ (adiponectin) is an adipose-derived plasma protein that functions in hepatic lipoprotein-lipid metabolism [70]. Several pieces of evidence indicate that diverse polymorphisms in ADIPOQ may increase susceptibility to NAFLD [70, 71]. One study reported that the ADIPOQ methylation rate in rats with NAFLD was higher than in control animals [72]. ADIPOQ in NAFLD patients is a risk factor for progression to liver cancer, and ADIPOQ is significantly decreased in patients with liver metastases [73].
Research has shown that the methylation rate of ADIPOQ in the NAFLD rat model is higher than in controls; further, alteration of the methylation rate pattern of ADIPOQ was hepatoprotective in the NAFLD group [72]. Another study reported that the level of ADIPOQ in serum is lower in NAFLD than controls and was associated with increased liver enzymes and lipid profile changes in patients with NAFLD [74]. Several studies have suggested that fenofibrate may modulate the level of adiponectin in diabetes, cardiomyocyte hypertrophy, and hypertriglyceridemia [75-77]. Fenofibrate caused an increase in serum adiponectin [78]. Fenofibrate may enhance adiponectin expression through modulation of PPAR-alpha expression [76]. Fenofibrate may also promote adiponectin through the AMPK signaling pathway [79]. Other researchers claimed that fenofibrate significantly reduced proinflammatory biomarkers and ameliorated adipocytokines through induction of adiponectin [80]. In our study, ADIPOQ was one of the highest scoring targets in terms of drug interactions with fenofibrate and an association with NAFLD.

LEP (leptin) is a polypeptide hormone that interacts with its receptor lepRb [81]. In a number of studies, the pathogenesis of leptin in NAFLD has been investigated. The level of leptin significantly increased in the serum of patients with NAFLD and in animal models of the disease and possibly normalized with the development of hepatocyte steatosis [81-84]. Leptin may be implicated in steatosis progression via activation of the PI3-K/Akt kinase pathway via OB-R $[85,86]$. Numerous studies have reported that fenofibrate affects LEP expression. Previous clinical studies have also shown that fenofibrate affects the level of leptin in patients with dyslipidemia and hypertriglyceridemia and improves insulin sensitivity [80, 87-89]. Furthermore, LEP scored 
highly in both the drug interaction and diseases-relation interrogation, scoring 0.829 and 0.4 , respectively.

SIRT1 is one of the important genes identified in the pathogenesis of fatty liver disease. SIRT1, a NADPHdependent deacetylase, has a vital function in cellular processes, including stress response, transcriptional regulation, longevity, and apoptosis [90]. A number of reports implicate miRNAs that target SIRT1 in the pathogenesis of NAFLD [91-93]. SIRT1 is significantly downregulated in NAFLD [94], and interventions aimed at modulating SIRT1 have shown positive effects on NAFLD [95-99]. Fenofibrate can indirectly upregulate SIRT1 and repress hepatocyte apoptosis via SIRT1 and FoxO1 [100, 101]. The upregulation of SIRT1 may be accomplished through AMPK in TNF- $\alpha$ stimulated adipocytes [102]. Another study showed that fenofibrate promotes SIRT1 expression, causing a reduction in NF- $\kappa$ B activity [103]. Fenofibrate has been shown to affect a reduction in fat deposition and to alleviate inflammation through SIRT1-dependent pathways [104, 105]. In our study, SIRT1 was identified as one of critical gene associations with NAFLD and exhibited a robust interaction with fenofibrate.

Apolipoprotein C3 (APOC3) is a small protein on the surface of lipoprotein particles and has a vital role in regulating triglyceride metabolism. APOC3 has a potent inhibitor effect on lipoprotein lipase [106]. A study by Pavia et al. indicated that overexpression of APOC3 results in pathological features in the liver similar to NAFLD such as inflammation, hepatocyte apoptosis, oxidative stress, and increased liver lipid content [107]. It has been reported that fenofibrate significantly reduces the level of APOC3. In this study, fenofibrate demonstrated a robust interaction with APOC3 based upon the STITCH score (high confidence: 0.944). APOC3 placed eleventh in the curated diseases database (Table 1), indicating it may have a role in the pathogenesis of NAFLD.

MMP (matrix metalloproteinase) is a proteinase that can degrade components of the extracellular matrix and diverse nonmatrix proteins. MMPs have been shown to be involved in the pathogenesis of liver diseases [108]. MMP1 may have a role in the progression of NAFLD to NASH and then to liver fibrosis [109, 110]. Two studies demonstrated that fenofibrate could decrease MMP1 and that it repressed the enzymic actions of MMP2 and MMP9 [57, 111]. MMP1, as demonstrated here, shows significant interaction with fenofibrate (high confidence score based on STITCH of 0.872).

SCARB1 (scavenger receptor class B, type I) is a highdensity lipoprotein (HDL) receptor that facilitates uptake of cholesterol (Cho) from HDL to hepatocytes [112, 113]. Recently, it has been suggested that SCARB1 may be associated with NAFLD [114] and several studies suggest that fenofibrate affects SCARB1 [115-117]. Those studies have proposed that fenofibrate may enhance the degradation of SCARB1 in a postendoplasmic reticulum or postplasma membrane compartment [115]. However, it is possible that fenofibrate does not directly inhibit SCARB1 [118]. The posttranscriptional regulation of fenofibrate may be dependent upon PPAR $\alpha$ expression [117]. According to our data shown in Table 1, SCARB1 is one of the top predicted targets for fenofibrate interaction, and investigation in the curated database revealed its relationship to NAFLD.

LDLR (low-density apolipoprotein receptor) is a mediator for cholesterol uptake in cells. It plays a crucial function in the clearance of cholesterol by the liver [119]. LDLR deficient rodents have been used to establish models of NAFLD $[120,121]$. In those models, elevations in hepatic neutral and hepatic proinflammatory oxylipins were observed [122]. Some patients with NAFLD have been found to have mutations in LDLR genes [123]. Numerous studies have also demonstrated that fenofibrate affects LDLR expression; fenofibrate elevated hepatic LDLR via Akt phosphorylation and maturation of SREBP2 [124]. As shown in Table 1, LDLR was one of the eleven important genes that interacted with fenofibrate and was associated with NAFLD.

VLDLR (very-low-density lipoprotein receptor) has a critical role in modulating serum triglycerides and NAFLD progression [125]. Research on a mouse NAFLD model has demonstrated that antagonism of $\operatorname{PPAR} \beta / \delta$ may regulate VLDLR and influence the serum triglyceride level and progression of NAFLD [125]. Studies have indicated that fenofibrate could influence VLDLR, but its mechanism and exact effect are still unclear $[126,127]$.

GSTP1 (glutathione S transferase Pi 1) is a gene that has a vital role in antioxidant defense through detoxifying foreign substances and inactivating byproducts of oxidative stress [128, 129]. Moreover, several studies published that some polymorphisms of GSTP1 are frequent in patients with NAFLD [130, 131]. The effect of fenofibrate on GSTP1 has not been studied in depth, and the available results are contradictory [132-136]. However, GSTP1 was one of eleven significant targets identified in our study and listed in Table 1.

\section{Conclusion}

In this study, we investigated the effect of fenofibrate on important targets in NAFLD. Our results indicate that fenofibrate may influence essential genes in NAFLD via an, as yet, undetermined mechanism. Fenofibrate could therefore benefit patients by preventing progression or even reversing severity of NAFLD. According to the results presented here, fenofibrate significantly influences essential biological pathways, including lipid metabolic processes via the PPAR signaling pathway and the AMPK signaling pathway. Notably, those targets have been validated as featuring in the pathogenesis of NAFLD. Consequently, fenofibrate may offer a significant benefit to patients with NAFLD, though further molecular and clinical investigation is required.

\section{Data Availability}

Data associated with this study are available from the authors upon a reasonable request.

\section{Conflicts of Interest}

The authors declare that they have no conflicts of interest. 


\section{References}

[1] V. Manne, P. Handa, and K. V. Kowdley, "Pathophysiology of nonalcoholic fatty liver disease/nonalcoholic steatohepatitis," Clinics in Liver Disease, vol. 22, no. 1, pp. 23-37, 2018.

[2] F. Mansour-Ghanaei, F. Joukar, S. N. Mobaraki, S. Mavaddati, S. Hassanipour, and M. Sepehrimanesh, "Prevalence of non-alcoholic fatty liver disease in patients with diabetes mellitus, hyperlipidemia, obesity and polycystic ovary syndrome: A cross- sectional study in north of Iran," Diabetes \& Metabolic Syndrome: Clinical Research \& Reviews, vol. 13, no. 2, pp. 1591-1596, 2019.

[3] G. Targher, C. D. Byrne, and H. Tilg, "NAFLD and increased risk of cardiovascular disease: clinical associations, pathophysiological mechanisms and pharmacological implications," Gut, vol. 69, no. 9, pp. 1691-1705, 2020.

[4] Z. M. Younossi, A. B. Koenig, D. Abdelatif, Y. Fazel, L. Henry, and M. Wymer, "Global epidemiology of nonalcoholic fatty liver disease-meta-analytic assessment of prevalence, incidence, and outcomes," Hepatology, vol. 64, no. 1, pp. 73-84, 2016.

[5] S. Sookoian and C. J. Pirola, "Precision medicine in nonalcoholic fatty liver disease: new therapeutic insights from genetics and systems biology," Clinical and Molecular Hepatology, vol. 26, no. 4, pp. 461-475, 2020.

[6] V. Nobili, A. Alisi, L. Valenti, L. Miele, A. E. Feldstein, and N. Alkhouri, "NAFLD in children: new genes, new diagnostic modalities and new drugs," Nature Reviews Gastroenterology \& Hepatology, vol. 16, no. 9, pp. 517-530, 2019.

[7] B. A. Neuschwander-Tetri, "Therapeutic landscape for NAFLD in 2020," Gastroenterology, vol. 158, no. 7, pp. 1984-1998.e3, 2020.

[8] M. Mahjoubin-Tehran, A. De Vincentis, D. P. Mikhailidis et al., "Non-alcoholic fatty liver disease and steatohepatitis: State of the art on effective therapeutics based on the gold standard method for diagnosis," Molecular Metabolism, vol. 50, p. 101049, 2021.

[9] M. Bagherniya, V. Nobili, C. N. Blesso, and A. Sahebkar, "Medicinal plants and bioactive natural compounds in the treatment of non-alcoholic fatty liver disease: A clinical review," Pharmacological Research, vol. 130, pp. 213-240, 2018.

[10] G. Ranjbar, D. P. Mikhailidis, and A. Sahebkar, "Effects of newer antidiabetic drugs on nonalcoholic fatty liver and steatohepatitis: Think out of the box!," Metabolism, vol. 101, p. 154001, 2019.

[11] Y. Sumida and M. Yoneda, "Current and future pharmacological therapies for NAFLD/NASH," Journal of Gastroenterology, vol. 53, no. 3, pp. 362-376, 2018.

[12] R. S. Rosenson, "Fenofibrate: treatment of hyperlipidemia and beyond," Expert Review of Cardiovascular Therapy, vol. 6, no. 10, pp. 1319-1330, 2008.

[13] M. Ruscica, N. Ferri, R. D. Santos, C. R. Sirtori, and A. Corsini, "Lipid lowering drugs: present status and future developments," Current Atherosclerosis Reports, vol. 23, no. 5, p. 17, 2021.

[14] A. Sahebkar and G. F. Watts, "New LDL-cholesterol lowering therapies: pharmacology, clinical trials, and relevance to acute coronary syndromes," Clinical Therapeutics, vol. 35, no. 8, pp. 1082-1098, 2013.

[15] A. Sahebkar and G. F. Watts, "New therapies targeting apoB metabolism for high-risk patients with inherited dyslipidae- mias: what can the clinician expect?," Cardiovascular Drugs and Therapy, vol. 27, no. 6, pp. 559-567, 2013.

[16] S. Kersten and R. Stienstra, "The role and regulation of the peroxisome proliferator activated receptor alpha in human liver," Biochimie, vol. 136, pp. 75-84, 2017.

[17] M. L. Morieri, H. S. Shah, J. Sjaarda et al., "PPARAPolymorphism influences the cardiovascular benefit of Fenofibrate in type 2 Diabetes: Findings From ACCORD-Lipid," Diabetes, vol. 69, no. 4, pp. 771-783, 2020.

[18] J. Oscarsson, K. Önnerhag, U. Risérus et al., "Effects of free omega-3 carboxylic acids and fenofibrate on liver fat content in patients with hypertriglyceridemia and non-alcoholic fatty liver disease: a double-blind, randomized, placebo-controlled study," Journal of Clinical Lipidology, vol. 12, no. 6, pp. 1390403.e4, 2018.

[19] A. Keech, R. J. Simes, P. Barter, J. Best, R. Scott, and M. R. Taskinen, "Effects of long-term fenofibrate therapy on cardiovascular events in 9795 people with type 2 diabetes mellitus (the FIELD study): randomised controlled trial," The Lancet, vol. 366, no. 9500, pp. 1849-1861, 2005.

[20] K. Esenboga, Ö. F. Çiçek, A. A. Oktay, P. Aribal Ayral, and A. Gürlek, "Effect of fenofibrate on serum nitric oxide levels in patients with hypertriglyceridemia," Advances in clinical and experimental medicine: official organ Wroclaw Medical University, vol. 28, no. 7, pp. 931-936, 2019.

[21] J. Zhang, Y. Cheng, J. Gu et al., "Fenofibrate increases cardiac autophagy via FGF21/SIRT1 and prevents fibrosis and inflammation in the hearts of Type 1 diabetic mice," Clinical Science, vol. 130, no. 8, pp. 625-641, 2016.

[22] J. Sun, Z. Zheng, Q. Chen, Y. Pan, M. Quan, and Y. Dai, "Fenofibrate potentiates chemosensitivity to human breast cancer cells by modulating apoptosis via AKT/NF-\&kappa;B pathway," OncoTargets and Therapy, vol. Volume 12, pp. 773-783, 2019.

[23] M. Botta, M. Audano, A. Sahebkar, C. R. Sirtori, N. Mitro, and M. Ruscica, "PPAR agonists and metabolic syndrome: An established role?," International Journal of Molecular Sciences, vol. 19, no. 4, p. 1197, 2018.

[24] G. Derosa, P. Maffioli, and A. Sahebkar, "Plasma uric acid concentrations are reduced by fenofibrate: a systematic review and meta-analysis of randomized placebocontrolled trials," Pharmacological Research, vol. 102, pp. 63-70, 2015.

[25] G. Derosa, A. Sahebkar, and P. Maffioli, "The role of various peroxisome proliferator-activated receptors and their ligands in clinical practice," Journal of Cellular Physiology, vol. 233, no. 1, pp. 153-161, 2018.

[26] A. Sahebkar, M. C. Serban, D. P. Mikhailidis et al., "Head-tohead comparison of statins versus fibrates in reducing plasma fibrinogen concentrations: a systematic review and metaanalysis," Pharmacological Research, vol. 103, pp. 236-252, 2016.

[27] A. Sahebkar and G. F. Watts, "Role of selective peroxisome proliferator-activated receptor modulators in managing cardiometabolic disease: tale of a roller-coaster," Diabetes, Obesity and Metabolism, vol. 16, no. 9, pp. 780-792, 2014.

[28] H. Yaribeygi, M. T. Mohammadi, R. Rezaee, and A. Sahebkar, "Fenofibrate improves renal function by amelioration of NOX-4, IL-18, and p53 expression in an experimental model of diabetic nephropathy," Journal of Cellular Biochemistry, vol. 119, no. 9, pp. 7458-7469, 2018. 
[29] S. M. El-Haggar and T. M. Mostafa, "Comparative clinical study between the effect of fenofibrate alone and its combination with pentoxifylline on biochemical parameters and liver stiffness in patients with non-alcoholic fatty liver disease," Hepatology International, vol. 9, no. 3, pp. 471-479, 2015.

[30] C. Fernández-Miranda, M. Pérez-Carreras, F. Colina, G. López-Alonso, C. Vargas, and J. A. Solís-Herruzo, “A pilot trial of fenofibrate for the treatment of non-alcoholic fatty liver disease," Digestive and Liver Disease, vol. 40, no. 3, pp. 200-205, 2008.

[31] J. Piñero, J. M. Ramírez-Anguita, J. Saüch-Pitarch et al., "The DisGeNET knowledge platform for disease genomics: 2019 update," Nucleic Acids Research, vol. 48, no. D1, pp. D845Dd55, 2019.

[32] S. Pletscher-Frankild, A. Pallejà, K. Tsafou, J. X. Binder, and L. J. Jensen, "DISEASES: Text mining and data integration of disease-gene associations," Methods, vol. 74, pp. 83-89, 2015.

[33] M. C. Sinton, D. C. Hay, and A. J. Drake, "Metabolic control of gene transcription in non-alcoholic fatty liver disease: the role of the epigenome," Clinical Epigenetics, vol. 11, no. 1, p. 104, 2019.

[34] M. Eslam, L. Valenti, and S. Romeo, "Genetics and epigenetics of NAFLD and NASH: clinical impact," Journal of Hepatology, vol. 68, no. 2, pp. 268-279, 2018.

[35] M. Yan, F. L. Meng, R. J. Lu, X. Q. Jia, and X. C. Zhao, “Therapy effects of fenofibrate on alcoholic fatty liver and druginduced fatty liver in rats," Zhonghua Gan Zang Bing Za Zhi, vol. 11, no. 2, pp. 86-89, 2003.

[36] J. N. van der Veen, S. Lingrell, X. Gao et al., "Fenofibrate, but not ezetimibe, prevents fatty liver disease in mice lacking phosphatidylethanolamine $N$-methyltransferase," Journal of Lipid Research, vol. 58, no. 4, pp. 656-667, 2017.

[37] M. Yaghoubi, S. Jafari, B. Sajedi et al., "Comparison of fenofibrate and pioglitazone effects on patients with nonalcoholic fatty liver disease," European Journal of Gastroenterology \& Hepatology, vol. 29, no. 12, pp. 1385-1388, 2017.

[38] A. Rogue, S. Anthérieu, A. Vluggens et al., "PPAR agonists reduce steatosis in oleic acid-overloaded HepaRG cells," Toxicology and Applied Pharmacology, vol. 276, no. 1, pp. 73-81, 2014.

[39] V. Dubois, J. Eeckhoute, P. Lefebvre, and B. Staels, "Distinct but complementary contributions of PPAR isotypes to energy homeostasis," The Journal of Clinical Investigation, vol. 127, no. 4, pp. 1202-1214, 2017.

[40] A. Z. Mirza, I. I. Althagafi, and H. Shamshad, "Role of PPAR receptor in different diseases and their ligands: physiological importance and clinical implications," European Journal of Medicinal Chemistry, vol. 166, pp. 502-513, 2019.

[41] D. P. Kumar, R. Caffrey, J. Marioneaux et al., "The PPAR $\alpha / \gamma$ agonist saroglitazar improves insulin resistance and steatohepatitis in a diet induced animal model of nonalcoholic fatty liver disease," Scientific Reports, vol. 10, no. 1, p. 9330, 2020.

[42] J. Boeckmans, A. Natale, M. Rombaut et al., "Anti-NASH drug development hitches a lift on PPAR agonism," Cells, vol. 9, no. 1, p. 37, 2020.

[43] M. J. Westerouen van Meeteren, J. P. H. Drenth, and E. Tjwa, "Elafibranor: a potential drug for the treatment of nonalcoholic steatohepatitis (NASH)," Expert Opinion on Investigational Drugs, vol. 29, no. 2, pp. 117-123, 2020.
[44] K. Motojima, P. Passilly, J. M. Peters, F. J. Gonzalez, and N. Latruffe, "Expression of Putative Fatty Acid Transporter Genes Are Regulated by Peroxisome Proliferator-activated Receptor $\alpha$ and $\gamma$ Activators in a Tissue- and Inducerspecific Manner," The Journal of Biological Chemistry, vol. 273, no. 27, pp. 16710-16714, 1998.

[45] P. Pettinelli and L. A. Videla, "Up-regulation of PPAR- $\gamma$ mRNA expression in the liver of obese patients: an additional reinforcing lipogenic mechanism to SREBP-1c induction," The Journal of Clinical Endocrinology and Metabolism, vol. 96, no. 5, pp. 1424-1430, 2011.

[46] M. Nakamuta, M. Kohjima, S. Morizono et al., "Evaluation of fatty acid metabolism-related gene expression in nonalcoholic fatty liver disease," International Journal of Molecular Medicine, vol. 16, no. 4, pp. 631-635, 2005.

[47] J. Skat-Rørdam, D. Højland Ipsen, J. Lykkesfeldt, and P. Tveden-Nyborg, "A role of peroxisome proliferatoractivated receptor $\gamma$ in non-alcoholic fatty liver disease," Basic \& Clinical Pharmacology \& Toxicology, vol. 124, no. 5, pp. 528-537, 2019.

[48] K. H. Liss and B. N. Finck, "PPARs and nonalcoholic fatty liver disease," Biochimie, vol. 136, pp. 65-74, 2017.

[49] Z. M. Younossi, L. Henry, H. Bush, and A. Mishra, "Clinical and economic burden of nonalcoholic fatty liver disease and nonalcoholic steatohepatitis," Clinics in Liver Disease, vol. 22, no. 1, pp. 1-10, 2018.

[50] E. Buzzetti, M. Pinzani, and E. A. Tsochatzis, "The multiple-hit pathogenesis of non-alcoholic fatty liver disease (NAFLD)," Metabolism, vol. 65, no. 8, pp. 10381048, 2016.

[51] S. Wu and M. H. Zou, "AMPK, Mitochondrial function, and cardiovascular disease," International journal of molecular sciences., vol. 21, no. 14, p. 4987, 2020.

[52] D. Garcia, K. Hellberg, A. Chaix et al., "Genetic liverspecific AMPK activation protects against diet-induced obesity and NAFLD," Cell Reports, vol. 26, no. 1, pp. 192-208.e6, 2019.

[53] W. Liang, A. L. Menke, A. Driessen et al., "Establishment of a general NAFLD scoring system for rodent models and comparison to human liver pathology," PloS One, vol. 9, no. 12, article e115922, 2014.

[54] S. Herzig and R. J. Shaw, "AMPK: guardian of metabolism and mitochondrial homeostasis," Nature Reviews Molecular Cell Biology, vol. 19, no. 2, pp. 121-135, 2018.

[55] B. K. Smith, K. Marcinko, E. M. Desjardins, J. S. Lally, R. J. Ford, and G. R. Steinberg, "Treatment of nonalcoholic fatty liver disease: role of AMPK," American Journal of Physiology Endocrinology and Metabolism, vol. 311, no. 4, pp. E730E740, 2016.

[56] T. A. Diniz, E. A. de Lima Junior, A. A. Teixeira et al., “Aerobic training improves NAFLD markers and insulin resistance through AMPK- PPAR- $\alpha$ signaling in obese mice," Life Sciences, vol. 266, article 118868, 2021.

[57] S. C. Tsai, M. H. Tsai, C. F. Chiu et al., "AMPK-dependent signaling modulates the suppression of invasion and migration by fenofibrate in CAL 27 oral cancer cells through NF$\kappa \mathrm{B}$ pathway," Environmental Toxicology, vol. 31, no. 7, pp. 866-876, 2016.

[58] C. E. Foulds, L. S. Treviño, B. York, and C. L. Walker, "Endocrine-disrupting chemicals and fatty liver disease," Nature Reviews Endocrinology, vol. 13, no. 8, pp. 445-457, 2017. 
[59] J. Han, E. Li, L. Chen et al., "The CREB coactivator CRTC2 controls hepatic lipid metabolism by regulating SREBP1," Nature, vol. 524, no. 7564, pp. 243-246, 2015.

[60] N. Ziamajidi, S. Khaghani, G. Hassanzadeh et al., "Amelioration by chicory seed extract of diabetes- and oleic acidinduced non- alcoholic fatty liver disease (NAFLD)/nonalcoholic steatohepatitis (NASH) via modulation of PPAR $\alpha$ and SREBP-1," Food and Chemical Toxicology: an international journal published for the British Industrial Biological Research Association, vol. 58, pp. 198-209, 2013.

[61] G. Fan, T. Shi, and S. Xiao, "Clinical investigation of relationship between SIRT3 and hepatocyte steatosis in nonalcoholic fatty liver disease," Chinese Journal of Gastroenterology, vol. 14, no. 5, pp. 261-265, 2009.

[62] Y. R. Kim, E. J. Lee, K. O. Shin et al., "Hepatic triglyceride accumulation via endoplasmic reticulum stress-induced SREBP-1 activation is regulated by ceramide synthases," Experimental \& Molecular Medicine, vol. 51, no. 11, pp. 116, 2019.

[63] J. P. Zhou, Y. D. Ren, Q. Y. Xu et al., “Obesity-induced upregulation of ZBTB7A promotes lipid accumulation through SREBP1," BioMed Research International, vol. 2020, Article ID 4087928, 13 pages, 2020.

[64] S. O. Lee, Y. Xu, H. Han et al., "Fermented rhus verniciflua stokes extract alleviates nonalcoholic fatty liver through the AMPK/SREBP1/PCSK9 pathway in HFD-induced nonalcoholic fatty liver animal model," Applied Sciences, vol. 10, no. 19, p. $6833,2020$.

[65] J. Hu, W. Hong, K. N. Yao, X. H. Zhu, Z. Y. Chen, and L. Ye, "Ursodeoxycholic acid ameliorates hepatic lipid metabolism in LO2 cells by regulating the AKT/mTOR/SREBP-1 signaling pathway," World Journal of Gastroenterology, vol. 25, no. 12, pp. 1492-1501, 2019.

[66] G. L. Wang, Y. C. Fu, W. C. Xu, Y. Q. Feng, S. R. Fang, and $\mathrm{X}$. H. Zhou, "Resveratrol inhibits the expression of SREBP1 in cell model of steatosis via Sirt1-FOXO1 signaling pathway," Biochemical and Biophysical Research Communications, vol. 380, no. 3, pp. 644-649, 2009.

[67] F. Yan, Q. Wang, C. Xu et al., "Peroxisome proliferatoractivated receptor $\alpha$ activation induces hepatic steatosis, suggesting an adverse effect," PLoS One, vol. 9, no. 6, 2014.

[68] T. L. Parry, G. Desai, J. C. Schisler et al., "Fenofibrate unexpectedly induces cardiac hypertrophy in mice lacking MuRF1," Cardiovascular Pathology: the official journal of the Society for Cardiovascular Pathology, vol. 25, no. 2, pp. 127-140, 2016.

[69] A. K. Min, J. Y. Jeong, Y. Go et al., "cAMP response element binding protein $\mathrm{H}$ mediates fenofibrate-induced suppression of hepatic lipogenesis," Diabetologia, vol. 56, no. 2, pp. 412422, 2013.

[70] B. F. Wang, Y. Wang, R. Ao, J. Tong, and B. Y. Wang, “AdipoQT45 G and G276 T polymorphisms and susceptibility to nonalcoholic fatty liver disease among Asian populations: a meta-analysis and meta-regression," Journal of Clinical Laboratory Analysis, vol. 30, no. 1, pp. 47-57, 2016.

[71] M. Liu, S. Liu, M. Shang et al., "Association between ADIPOQ G276T and C11377G polymorphisms and the risk of non-alcoholic fatty liver disease: An updated meta-analysis," Molecular Genetics \& Genomic Medicine, vol. 7, no. 5, article e624, 2019.

[72] W. J. Chen, B. Cai, H. T. Chen et al., "The role ofADIPOQmethylation in curcumin-administrated experimental nonal- coholic fatty liver disease," Journal of Digestive Diseases, vol. 17, no. 12, pp. 829-836, 2016.

[73] R. Divella, A. Daniele, R. A. F. F. A. E. L. E. de Luca et al., "Synergism of adipocytokine profile andADIPOQ/TNF- $\alpha$ Polymorphisms in NAFLD-associated mets predict colorectal liver metastases outgrowth," Cancer Genomics \& Proteomics, vol. 16, no. 6, pp. 519-530, 2019.

[74] A. A. Mahmoud, H. M. Moghazy, L. M. Yousef, and A. N. Mohammad, "Adiponectin rs2241766 and rs266729 gene polymorphisms in non-alcoholic fatty liver disease," Gene Reports, vol. 15, p. 100381, 2019.

[75] K. K. Koh, M. J. Quon, K. C. Shin et al., "Significant differential effects of omega-3 fatty acids and fenofibrate in patients with hypertriglyceridemia," Atherosclerosis, vol. 220, no. 2, pp. 537-544, 2012.

[76] H. L. Jen, W. H. Yin, J. W. Chen, and S. J. Lin, "Endothelin-1induced cell hypertrophy in cardiomyocytes is improved by fenofibrate: possible roles of adiponectin," Journal of Atherosclerosis and Thrombosis, vol. 24, no. 5, pp. 508-517, 2017.

[77] Y. J. Hsu, L. C. Wang, W. S. Yang, C. M. Yang, and C. H. Yang, "Effects of Fenofibrate on Adiponectin Expression in Retinas of Streptozotocin- Induced Diabetic Rats," Journal of Diabetes Research, vol. 2014, Article ID 540326, 14 pages, 2014

[78] K. Oki, J. Koide, S. Nakanishi, R. Nakashima, and K. Yamane, "Fenofibrate increases high molecular weight adiponectin in subjects with hypertriglyceridemia," Endocrine Journal, vol. 54, no. 3, pp. 431-435, 2007.

[79] P. Li, R. Shibata, S. Maruyama et al., "Fenofibrate promotes ischemia-induced revascularization through the adiponectin-dependent pathway," American Journal of Physiology. Endocrinology and Metabolism, vol. 299, no. 4, pp. E560-E566, 2010.

[80] K. K. Koh, M. J. Quon, S. Lim et al., "Effects of fenofibrate therapy on circulating adipocytokines in patients with primary hypertriglyceridemia," Atherosclerosis, vol. 214, no. 1, pp. 144-147, 2011.

[81] M. Swellam and N. Hamdy, "Association of nonalcoholic fatty liver disease with a single nucleotide polymorphism on the gene encoding leptin receptor," IUBMB Life, vol. 64, no. 2, pp. 180-186, 2012.

[82] Q. Zhang, J. Wang, F. Huang, Y. Yao, and L. Xu, "Leptin induces NAFLD progression through infiltrated CD8+ T lymphocytes mediating pyroptotic-like cell death of hepatocytes and macrophages," Digestive and Liver Disease, vol. 53, no. 5, pp. 598-605, 2021.

[83] V. Marques, M. B. Afonso, N. Bierig et al., "Adiponectin, leptin, and IGF-1 are useful diagnostic and stratification biomarkers of NAFLD," Frontiers in Medicine, vol. 8, 2021.

[84] L. Rotundo, A. Persaud, M. Feurdean, S. Ahlawat, and H. S. Kim, "The association of leptin with severity of nonalcoholic fatty liver disease: a population-based study," Clinical and Molecular Hepatology, vol. 24, no. 4, pp. 392-401, 2018.

[85] D. Xu, X. D. Huang, H. S. Luo, J. P. Yuan, H. Zhang, and $\mathrm{J}$. $\mathrm{Wu}$, "Impaired activation of phosphatidylinositol 3-kinase by leptin in NAFLD: a novel mechanism of hepatic leptin resistance," World Chinese Journal of Digestology, vol. 20, no. 32, pp. 3095-3100, 2012.

[86] D. Xu, X. D. Huang, J. P. Yuan et al., "Impaired activation of phosphatidylinositol 3-kinase by leptin is a novel mechanism 
of hepatic leptin resistance in NAFLD," Hepato-Gastroenterology, vol. 58, no. 110-111, pp. 1703-1707, 2011.

[87] L. Buldak, A. Dulawa-Buldak, K. Labuzek, and B. Okopien, "Effects of 90-day hypolipidemic treatment on insulin resistance, adipokines and proinflammatory cytokines in patients with mixed hyperlipidemia and impaired fasting glucose," International Journal of Clinical Pharmacology and Therapeutics, vol. 50, no. 11, pp. 805-813, 2012.

[88] M. Florentin, E. N. Liberopoulos, C. C. Tellis, C. S. Derdemezis, M. Elisaf, and A. Tselepis, "Effects of rimonabant, as monotherapy and in combination with fenofibrate or ezetimibe, on plasma adipokine levels: a pilot study," Angiology, vol. 61, no. 4, pp. 365-371, 2010.

[89] T. Damci, S. Tatliagac, Z. Osar, and K. Ilkova, "Fenofibrate treatment is associated with better glycemic control and lower serum leptin and insulin levels in type 2 diabetic patients with hypertriglyceridemia," European Journal of Internal Medicine, vol. 14, no. 6, pp. 357-360, 2003.

[90] H. S. Ghosh, J. V. Spencer, B. Ng, M. W. McBurney, and P. D. Robbins, "Sirt1 interacts with transducin-like enhancer of split-1 to inhibit nuclear factor $\kappa \mathrm{B}$-mediated transcription," The Biochemical Journal, vol. 408, no. 1, pp. 105-111, 2007.

[91] L. Wang, M. Sun, Y. Cao et al., "miR-34a regulates lipid metabolism by targeting SIRT1 in non-alcoholic fatty liver disease with iron overload," Archives of Biochemistry and Biophysics, vol. 695, p. 108642, 2020.

[92] Y. Wang, K. Zhu, W. Yu et al., "MiR-181b regulates steatosis in nonalcoholic fatty liver disease via targeting SIRT1," Biochemical and Biophysical Research Communications, vol. 493, no. 1, pp. 227-232, 2017.

[93] J. K. Long, W. Dai, Y. W. Zheng, and S. P. Zhao, "MiR-122 promotes hepatic lipogenesis via inhibiting the LKB1/AMPK pathway by targeting Sirt1 in non-alcoholic fatty liver disease," Molecular Medicine, vol. 25, no. 1, 2019.

[94] X. Q. Deng, L. L. Chen, and N. X. Li, "The expression of SIRT1 in nonalcoholic fatty liver disease induced by highfat diet in rats," Liver International, vol. 27, no. 5, pp. 708715, 2007.

[95] Y. Zhu, R. Liu, Z. Shen, and G. Cai, "Combination of luteolin and lycopene effectively protect against the "two-hit" in NAFLD through Sirt1/AMPK signal pathway," Life Sciences, vol. 256, article 117990, 2020.

[96] H. Nikroo, S. R. A. Hosseini, M. Fathi, M. A. Sardar, and M. Khazaei, "The effect of aerobic, resistance, and combined training on PPAR- $\alpha$, SIRT1 gene expression, and insulin resistance in high-fat diet-induced NAFLD male rats," Physiology \& Behavior, vol. 227, p. 113149, 2020.

[97] S. Li, Q. Qian, N. Ying et al., "Activation of the AMPK-SIRT1 pathway contributes to protective effects of Salvianolic acid a against lipotoxicity in hepatocytes and NAFLD in mice," Frontiers in Pharmacology, vol. 11, 2020.

[98] C. C. Chyau, H. F. Wang, W. J. Zhang et al., “Antrodan alleviates high-fat and high-fructose diet-induced fatty liver disease in C57BL/6 mice model via AMPK/Sirt1/SREBP-1c/ PPAR $\gamma$ pathway," International Journal of Molecular Sciences, vol. 21, no. 1, p. 360, 2020.

[99] H. Yao, X. Tao, L. Xu et al., "Dioscin alleviates non-alcoholic fatty liver disease through adjusting lipid metabolism via SIRT1/AMPK signaling pathway," Pharmacological Research, vol. 131, pp. 51-60, 2018.
[100] W. Wang, L. Bai, H. Qiao et al., "The protective effect of fenofibrate against TNF- $\alpha$-induced CD40 expression through SIRT1-mediated deacetylation of NF- $\kappa$ B in endothelial cells," Inflammation, vol. 37, no. 1, pp. 177-185, 2014.

[101] W. R. Wang, E. Q. Liu, J. Y. Zhang et al., “Activation of PPAR alpha by fenofibrate inhibits apoptosis in vascular adventitial fibroblasts partly through SIRT1-mediated deacetylation of FoxO1," Experimental Cell Research, vol. 338, no. 1, pp. 5463, 2015

[102] W. Wang, Q. Lin, R. Lin et al., "PPAR $\alpha$ agonist fenofibrate attenuates TNF- $\alpha$-induced CD40 expression in 3T3-L1 adipocytes via the SIRT1-dependent signaling pathway," Experimental Cell Research, vol. 319, no. 10, pp. 1523-1533, 2013.

[103] N. Chen, K. Jiang, and G. G. Yan, "Effect of fenofibrate on diabetic retinopathy in rats via SIRT1/NF- $\kappa$ B signaling pathway," European Review for Medical and Pharmacological Sciences, vol. 23, no. 19, pp. 8630-8636, 2019.

[104] S. Zhao, J. Li, N. Wang et al., "Fenofibrate suppresses cellular metabolic memory of high glucose in diabetic retinopathy via a sirtuin 1-dependent signalling pathway," Molecular Medicine Reports, vol. 12, no. 4, pp. 6112-6118, 2015.

[105] M. Jin, T. Zhu, D. R. Tocher et al., "Dietary fenofibrate attenuated high-fat-diet-induced lipid accumulation and inflammation response partly through regulation of ppar $\alpha$ and sirt1 in juvenile black seabream (Acanthopagrus schlegelii)," Developmental and Comparative Immunology, vol. 109, p. 103691, 2020.

[106] E. Christopoulou, V. Tsimihodimos, T. Filippatos, and M. Elisaf, "Apolipoprotein CIII and diabetes. Is there a link?," Diabetes/Metabolism Research and Reviews, vol. 35, no. 3, article e3118, 2019.

[107] A. A. Paiva, H. F. Raposo, A. C. Wanschel, T. R. Nardelli, and H. C. Oliveira, "Apolipoprotein CIII overexpression-induced hypertriglyceridemia increases nonalcoholic fatty liver disease in association with inflammation and cell death," Oxidative Medicine and Cellular Longevity, vol. 2017, Article ID 1838679, 18 pages, 2017.

[108] A. Naim, Q. Pan, and M. S. Baig, "Matrix metalloproteinases (MMPs) in liver diseases," Journal of Clinical and Experimental Hepatology, vol. 7, no. 4, pp. 367-372, 2017.

[109] E. H. Meng, J. M. Zhao, S. S. Wang et al., "Expression of matrix metalloproteinases in liver tissues with nonalcoholic steatohepatitis," World Chinese Journal of Digestology, vol. 10, no. 11, pp. 1257-1260, 2002.

[110] A. A. Mahmoud, A. S. Bakir, and S. S. Shabana, "Serum TGF$\beta$, serum MMP-1, and HOMA-IR as non-invasive predictors of fibrosis in Egyptian patients with NAFLD," Saudi Journal of Gastroenterology: official journal of the Saudi Gastroenterology Association, vol. 18, no. 5, pp. 327-333, 2012.

[111] K. S. Kim, D. H. Oh, H. M. Choi et al., "Pyrrolidine dithiocarbamate, a NF- $\kappa \mathrm{B}$ inhibitor, upregulates MMP-1 and MMP13 in IL- $1 \beta$-stimulated rheumatoid arthritis fibroblast-like synoviocytes," European Journal of Pharmacology, vol. 613, no. 1-3, pp. 167-175, 2009.

[112] X. Zhang and C. Fernández-Hernando, "The Janus-faced role of SR-BI in atherosclerosis," Nature Metabolism, vol. 1, no. 6, pp. 586-587, 2019.

[113] A. Helgadottir, P. Sulem, G. Thorgeirsson et al., "Rare SCARB1 mutations associate with high-density lipoprotein cholesterol but not with coronary artery disease," European Heart Journal, vol. 39, no. 23, pp. 2172-2178, 2018. 
[114] M. Tan, J. Ye, M. Zhao, X. Ke, K. Huang, and H. Liu, "Recent developments in the regulation of cholesterol transport by natural molecules," Phytotherapy Research, vol. 35, no. 10, pp. 5623-5633, 2021.

[115] D. Lan and D. L. Silver, "Fenofibrate Induces a Novel Degradation Pathway for Scavenger Receptor B-I Independent of PDZK1," The Journal of Biological Chemistry, vol. 280, no. 24, pp. 23390-23396, 2005.

[116] U. Panzenboeck, I. Kratzer, A. Sovic et al., "Regulatory effects of synthetic liver X receptor- and peroxisome-proliferator activated receptor agonists on sterol transport pathways in polarized cerebrovascular endothelial cells," The International Journal of Biochemistry \& Cell Biology, vol. 38, no. 8, pp. 1314-1329, 2006.

[117] P. Mardones, A. Pilon, M. Bouly et al., "Fibrates Downregulate Hepatic Scavenger Receptor Class B Type I Protein Expression in Mice," The Journal of Biological Chemistry, vol. 278 , no. 10 , pp. 7884-7890, 2003.

[118] T. J. Nieland, J. T. Shaw, F. A. Jaipuri et al., "Influence of HDL-cholesterol-elevating drugs on the in vitro activity of the HDL receptor SR-BI," Journal of Lipid Research, vol. 48, no. 8, pp. 1832-1845, 2007.

[119] M. Floeth, S. Elges, J. Gerss et al., "Low-density lipoprotein receptor (LDLR) is an independent adverse prognostic factor in acute myeloid leukaemia," British Journal of Haematology, vol. 192, no. 3, pp. 494-503, 2021.

[120] L. Álvarez-Amor, A. L. Sierra, A. Cárdenas et al., "Extra virgin olive oil improved body weight and insulin sensitivity in high fat diet-induced obese LDLr-/-.Leiden mice without attenuation of steatohepatitis," Scientific Reports, vol. 11, no. 1, 2021.

[121] M. H. Schoemaker, R. Kleemann, M. C. Morrison et al., "A casein hydrolysate based formulation attenuates obesity and associated nonalcoholic fatty liver disease and atherosclerosis in LDLr-/-.Leiden mice," PLoS One, vol. 12, no. 7, article e0180648, 2017.

[122] L. Li, G. F. Zhang, K. Lee et al., "A Western diet induced NAFLD in $\mathrm{LDLR}^{-1}$ - mice is associated with reduced hepatic glutathione synthesis," Free Radical Biology \& Medicine, vol. 96, pp. 13-21, 2016.

[123] E. Vilar-Gomez, S. Gawrieh, T. Liang, A. D. McIntyre, R. A. Hegele, and N. Chalasani, "Interrogation of selected genes influencing serum LDL-cholesterol levels in patients with well characterized NAFLD," Journal of Clinical Lipidology, vol. 15, no. 2, pp. 275-291, 2021.

[124] Z. Huang, X. Zhou, A. C. Nicholson, A. M. Gotto Jr., D. P. Hajjar, and J. Han, "Activation of peroxisome proliferatoractivated receptor- $\alpha$ in mice induces expression of the hepatic low-density lipoprotein receptor," British Journal of Pharmacology, vol. 155, no. 4, pp. 596-605, 2008.

[125] M. Zarei, E. Barroso, X. Palomer et al., "Pharmacological PPAR $\beta / \delta$ activation upregulates VLDLR in hepatocytes," Clínica e Investigación en Arteriosclerosis (English Edition), vol. 31, no. 3, pp. 111-118, 2019.

[126] S. Raza-Iqbal, T. Tanaka, M. Anai et al., "Transcriptome analysis of K-877 (a novel selective PPAR $\alpha$ modulator $(\mathrm{SPPARM} \alpha)$ )-regulated genes in primary human hepatocytes and the mouse liver," Journal of Atherosclerosis and Thrombosis, vol. 22, no. 8, pp. 754-772, 2015.

[127] Y. Gao, W. Shen, B. Lu, Q. Zhang, Y. Hu, and Y. Chen, "Upregulation of hepatic VLDLR via PPAR $\alpha$ is required for the triglyceride- lowering effect of fenofibrate," Journal of Lipid Research, vol. 55, no. 8, pp. 1622-1633, 2014.

[128] Q. Qadri, A. S. Sameer, Z. A. Shah et al., "Genetic polymorphism of the glutathione-S-transferase P1 gene (GSTP1) and susceptibility to prostate cancer in the Kashmiri population," Genetics and Molecular Research, vol. 10, no. 4, pp. 3038-3045, 2011.

[129] M. Hori, K. Oniki, T. Nakagawa et al., “Association between combinations of glutathione-S-transferaseM1,T1andP1genotypes and non-alcoholic fatty liver disease," Liver International, vol. 29, no. 2, pp. 164-168, 2009.

[130] V. P. Prysyazhnyuk, Z. I. Rossokha, and N. G. Gorovenko, "Variation in particular biochemical indicators, cytokine and adipokine profiles of the blood, and the structural and functional parameters of the liver in patients with nonalcoholic fatty liver disease and different genotypes by the polymorphic locus A313G of the GSTP1 gene," Cytology and Genetics, vol. 51, no. 6, pp. 455-461, 2017.

[131] M. Hashemi, E. Eskandari-Nasab, A. Fazaeli et al., "Association of genetic polymorphisms of glutathione-s-transferase genes (GSTT1,GSTM1, andGSTP1) and susceptibility to nonalcoholic fatty liver disease in Zahedan, Southeast Iran," DNA and Cell Biology, vol. 31, no. 5, pp. 672-677, 2012.

[132] J. Nishimura, Y. Dewa, T. Okamura et al., "Possible involvement of oxidative stress in fenofibrate-induced hepatocarcinogenesis in rats," Archives of Toxicology, vol. 82, no. 9, pp. 641-654, 2008.

[133] J. Nishimura, Y. Dewa, T. Okamura et al., "Role of Nrf2 and oxidative stress on fenofibrate-induced hepatocarcinogenesis in rats," Toxicological Sciences, vol. 106, no. 2, pp. 339-349, 2008.

[134] A. Nagyova, K. Raslova, Z. Kovacikova, V. Mongiellova, and E. Ginter, "Glutathione and glutathione-dependent enzymes in patients with hyperlipoproteinaemia treated with micronized fenofibrate," Klinicka Biochemie a Metabolismus, vol. 4, no. 4, pp. 242-244, 1996.

[135] M. Roques, D. Bagrel, J. Magdalou, and G. Siest, "Expression of arylhydrocarbon hydroxylase, epoxide hydrolases, glutathione S-transferase and UDP-glucuronosyltransferases in H5-6 hepatoma cells," General Pharmacology: The Vascular System, vol. 22, no. 4, pp. 677-684, 1991.

[136] A. Foliot, D. Touchard, and L. Mallet, "Inhibition of liver glutathione $S$-transferase activity in rats by hypolipidemic drugs related or unrelated to clofibrate," Biochemical Pharmacology, vol. 35, no. 10, pp. 1685-1690, 1986. 